\title{
The carbapenems issue
}

\author{
David Sotello MD, Wadih Chakkour MD, Kristen Fuhrmann Pharm D
}

\begin{abstract}
The development of antibiotics remains one of the great advances in medicine. Antibiotics have saved countless lives. Unfortunately, the widespread use of antimicrobials has led to the development of antimicrobial resistance. Antibiotic resistance is an important concern for public health; it is associated with poor outcomes. Carbapenems, members of the $\beta$-lactam class of antibiotics, have the broadest spectrum of antimicrobial activity. Carbapenem resistance is one of the toughest challenges in infectious diseases; it is associated with high mortality and is seen more often now due to the proliferation of multi-drug resistant bacteria. Multiple genes that cause carbapenem resistance have been identified. Resistance transmission is usually nosocomial, but community-acquired infections with resistance have been reported. Early recognition of high risk patients for multi-drug resistant infections is fundamental for adequate management. The rational use of antibiotics is required to prevent the spread of antimicrobial resistance; this requires multidisciplinary efforts among clinicians, Infection Control departments, and Antimicrobial Stewardship programs.
\end{abstract}

Keywords: Resistance, Antibiotics, Carbapenem, Carbapenemase, $\beta$-lactamase

Antibiotic resistance is a matter of the highest concern for worldwide public health. The correct use of antibiotics has saved millions of lives since Fleming's penicillin discovery in the 1920 s. ${ }^{1}$ It is no news that the widespread use and abuse of antibiotics have caused a new problem, namely resistance. Antibiotic resistance has been associated with at least 25,000 deaths/ year in the United States and costs more than $\$ 20$ billion in additional medical expenses every year., ${ }^{1,2}$ This problem has driven scientists and physicians to develop and use more antibiotics with different antimicrobial activity, but resistance is just a matter of time. Medical centers deal daily with some of the toughest challenges in infectious diseases, i.e., carbapenem

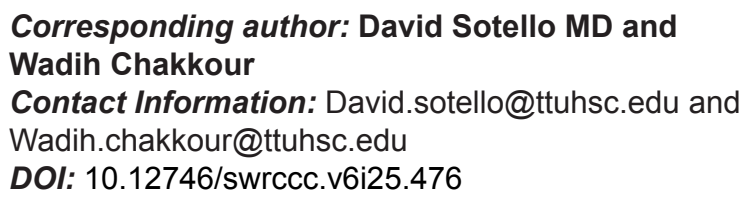

resistance. Carbapenems are the broadest spectrum antibiotics, previously considered the last line resource in antibiotic therapy, but some clinicians now use them routinely as first or second line antibiotic choices. This scenario can only result in more resistance.

Carbapenems, members of the $\beta$-lactam class of antibiotics that kill bacteria by binding to penicillin-binding proteins and inhibiting cell wall synthesis, were first used in the United States in the 1980s to treat resistant bacteria, mainly extended spectrum $\beta$-lactamases (ESBL) producing bacteria. They are used in a wide variety of infections that include bacteremia, intra-abdominal and pelvic infections, meningitis, pneumonia, urinary tract infections, soft tissue infections, osteomyelitis, etc. ${ }^{3}$ Carbapenems are used to treat most infections caused by Gramnegative and anaerobic bacteria, and they also have excellent Gram-positive coverage, including oxacillinsusceptible Staphylococcus aureus, but they lack activity against Stenotrophomonas maltophilia and 
Enterococcus faecium. ${ }^{3}$ Ertapenem lacks coverage against $P$ seudomonas aeruginosa and Acinetobacter baumanii. ${ }^{3}$ Meropenem and imipenem have excellent central nervous system (CNS) penetration, although imipenem has the highest risk for seizures. ${ }^{4}$ There is a lack of data about CNS penetration for doripenem and ertapenem. ${ }^{4}$ Dose adjustment may be required for renal impairment but not for liver impairment.

Due to widespread use of carbapenem, especially since the 2000 s, there has been an alarming surge of carbapenem resistance worldwide. In a recent European study, carbapenem resistance was reported in up to $7 \%$ of all the Klebsiella pneumoniae bloodstream isolates. ${ }^{1.5}$ This alarming increase in antimicrobial resistance mandates the development of a rational approach to antibiotic use. At the University Medical Center, Lubbock, Texas, there has been an increase in the incidence of carbapenem resistance. Recently, 16 cases of Pseudomonas aeruginosa with Verona integron-encoded metallo- $\beta$-lactamase (VIM), a type of carbapenemase not usually found in the United States, were identified. Previous outbreaks have occurred in the United States. The first outbreak was reported in $2005 .^{6}$

Carbapenem resistant infections have been reported to have an associated mortality rate between $40-80 \%$. $^{1,5}$ Carbapenem resistance transmission is usually plasmid-related, although chromosomal transmission has also been well documented. Different genes have been identified, including Klebsiella pneumoniae carbapenemase or bla KPC $_{2}$ (worldwide spread), New Delhi metallo- $\beta$-lactamase

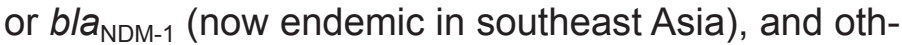
ers like OXA-48, VIM (usually found in Europe, Asia and South America), Imipenemase-1, etc. ${ }^{1,5,7}$

Carbapenem resistance transmission is usually nosocomial, but it has also been reported in the community, which represents a serious public health matter. ${ }^{7}$ The critical question is how to prevent further spread of carbapenem resistance. The first step is to identify the risk factors for carbapenem resistance and other multi-drug resistant (MDR) organisms, which include a recent admission to an acute care facility, prior admission to a long-term care facility, history of dialysis or chemotherapy in the last 12 months, previously known infection or carriage ${ }^{8}$ immune suppression, recent antimicrobial exposure, admission to an intensive care unit, and previous solid-organ or bone marrow transplant. ${ }^{9}$ These patients should be handled with strict infectious control measures to avoid further MDR spread. Hospital antimicrobial stewardship programs may also have an important role in the judicious use of antimicrobials.

The second crucial question is how to treat these infections. Currently, there are no guidelines regarding appropriate antimicrobial management of these patients, and as previously mentioned the mortality is high. Tigecycline, polymyxins, and aminoglycosides have been used, ${ }^{1}$ as well as anecdotal reports of double carbapenem coverage..$^{10}$ In the last few years, the development of newer antibiotic combinations like ceftazidime-avibactam and ceftolazone-tazobactam have been successfully used for these infections. ${ }^{1}$ More recently, newer drugs have been approved by the Food and Drug Administration or have been submitted for approval to manage these multidrug resistant infections (e.g., meropenem-vaborbactam, aztreonam-avibactam, plazomicin, cilastin-imipenem, eravacycline).

We recommend reserving carbapenems use to situations in which cultures shows bacteria resistant to all other antibiotics, in critically-ill patients known to be colonized with MDR bacteria, or in patients with high-risk factors for MDR infections. It is also important to differentiate between simple colonization and true infection such as in elderly patients with chronic Foley catheters. These patients have been exposed to multiple antibiotics and often grow ESBL organisms in urine that may not require treatment. Treatment should be reserved for symptomatic patients, i.e., presence of fever, abdominal pain, and/or altered mental status. Simple cystitis may be adequately treated by changing the Foley catheter and using nitrofurantoin or other antibiotics with a narrow spectrum.

Due to the associated mortality, prevention and management of carbapenem-resistant Enterobacteriaceae infections should be a high priority in hospitals and long-term health facilities. Antimicrobial stewardship may be a key element in the prevention of development of local resistance both in inpatient and outpatient settings. Infection control is crucial in containing imported 
cases from other facilities. Judicious use of carbapenems is also fundamental to prevent development of local resistance; these agents should be reserved to high-risk patients for MDR infections. Once a carbapenem-resistant Enterobacteriaceae infection is documented, infectious diseases consultation to assist in infection management is recommended.

Article citation: Sotello D, Chakkour W, Fuhrmann K. The Carbapenems Issue. The Southwest Respiratory and Critical Care Chronicles 2018; 6(25):5-7

From: Division of Pulmonary and Critical Care Medicine (DS, WC), Texas Tech University Health Sciences Center, Lubbock, Texas; Infectious Diseases pharmacist (KF) at University Medical Center, Lubbock, Texas.

Submitted: $1 / 6 / 2018$

Accepted: $4 / 27 / 2018$

Reviewer: Victor Test MD

Conflicts of interest: none

This work is licensed under a Creative Commons Attribution-ShareAlike 4.0 International License.

\section{REFERENCES}

1. Martens E, Demain AL. The antibiotic resistance crisis, with a focus on the United States. J Antibiot (Tokyo). 2017;70: $520-526$.
2. Marston HD, Dixon DM, Knisely JM, et al. Antimicrobial resistance. JAMA. 2016;316:1193-1204.

3. Doi Y, Chambers HF. Other $\beta$-Lactam Antibiotics. In: Mandell, Douglas, and Bennett's Principles and Practice of Infectious Diseases, Updated edition. 8th Edition, Pennsylvania: Saunders, 2015. Chapter 22:293-297.

4. Nau R, Sörgel F, Eiffert H. Penetration of drugs through the blood-cerebrospinal fluid/blood-brain barrier for treatment of central nervous system infections. Clin Microbiol Rev 2010;23:858-83.

5. Friedman ND, Carmeli Y, Walton AL, et al. Carbapenemresistant Enterobacteriaceae: a strategic roadmap for infection control. Infect Control Hosp Epidemiol 2017;38: 580-594.

6. Lolans K, Queenan AM, Bush K, et al. First nosocomial outbreak of Pseudomonas aeruginosa producing an integron-borne metallo-beta-lactamase (VIM-2) in the United States. Antimicrob Agents Chemother 2005;49:3538-40.

7. Kelly AM, Mathema B, Larson EL. Carbapenem-resistant Enterobacteriaceae in the community: a scoping review. See comment in PubMed Commons belowInt J Antimicrob Agents 2017;50:127-134.

8. Magiorakos AP, Burns K, Rodríguez Baño J, et al. Infection prevention and control measures and tools for the prevention of entry of carbapenem-resistant Enterobacteriaceae into healthcare settings: guidance from the European Centre for Disease Prevention and Control. Antimicrob Resist Infect Control 2017; 6: 113.

9. Codjoe FS, Donkor ES. Carbapenem resistance: a review. Med Sci (Basel) 2017;21:6(1).

10. Doi Y, Paterson DL. Carbapenemase-producing Enterobacteriaceae. Semin Respir Crit Care Med 2015;36:74-84. 